\title{
A utilização de pinos de fibra de vidros anatômicos como uma alternativa para a melhoria estética em tratamentos reabilitadores: revisão de literatura
}

\author{
The use of anatomical fiberglass posts as an alternative for an aesthetic improvement in
} rehabilitative treatments: literature review

El uso de postes anatómicos de fibra de vidrio como alternativa para una mejora estética en tratamientos rehabilitadores: revisión de la literatura

Recebido: 03/11/2021 | Revisado: 10/11/2021 | Aceito: 10/11/2021 | Publicado: 15/11/2021

\author{
Manuela Moraes Meireles \\ ORCID: https://orcid.org/0000-0001-9593-4663 \\ Centro Universitário Fametro, Brasil \\ E-mail: manu_moraes_meireles@hotmail.com \\ Neusimar Queiroz Coelho \\ ORCID: https://orcid.org/0000-0001-8758-8078 \\ Centro Universitário Fametro, Brasil \\ E-mail: coelhoneusimar@gmail.com \\ Gabriel Catunda de Souza \\ ORCID: https://orcid.org/0000-0002-0806-9841 \\ Centro Universitário Fametro, Brasil \\ E-mail: gabriel.catunda@fametro.edu.br
}

\begin{abstract}
Resumo
Introdução: Na odontologia são apresentados vários desafios e a reabilitação estética e funcional em dentes com destruição coronária é um deles. Objetivo Geral: elucidar uma revisão de literatura a respeito da reabilitação estética e funcional com pinos de fibra anatômicos. Metodologia: Para a construção desse artigo científico foi utilizada pesquisa de revisão bibliográfica descritiva da literatura. Resultados e Discussão: Quando apresentado casos onde existe uma grande perda na estrutura dentária, a utilização de pinos de fibra de vidro se apresenta como uma ótima alternativa oferecendo melhor suporte para reabilitar a unidade. Conclusão: concluímos que a técnica utilizada traz benefícios concretos tanto para o profissional quanto para o paciente. Por possuir o modulo de elasticidade próximo a dentina, possibilitando assim uma melhor distribuição de cargas, reduzindo assim o risco de fraturas.
\end{abstract}

Palavras-chave: Reabilitação; Pinos de fibra de vidro; Estética; Pinos anatômicos.

\begin{abstract}
Introduction: In dentistry there are several challenges and an aesthetic and functional rehabilitation of teeth with coronary destruction is one of them. General Objective: to elucidate a literature review regarding esthetic and functional rehabilitation with anatomical fiber pins. Methodology: For the construction of this scientific article, a descriptive bibliographic review of the literature was used. Results and Discussion: When presented cases where there is a great loss in the tooth structure, the use of fiberglass posts is presented as an excellent alternative offering better support to rehabilitate the unit. Conclusion: we conclude that the technique used brings concrete benefits for both the professional and the patient. Because it has a modulus of elasticity close to dentin, thus enabling a better distribution of loads, as well as the risk of fractures.
\end{abstract}

Keywords: Rehabilitation; Fiberglass pins; Aesthetics; Anatomical pins.

\section{Resumen}

Introducción: En odontología se presentan varios retos y la rehabilitación estética y funcional de dientes con destrucción coronaria es uno de ellos. Objetivo general: dilucidar una revisión de la literatura sobre rehabilitación estética y funcional con alfileres de fibra anatómicos. Metodología: Para la construcción de este artículo científico se utilizó una investigación de revisión descriptiva de la literatura. Resultados y Discusión: Cuando se presentan casos donde existe una gran pérdida en la estructura dentaria, el uso de postes de fibra de vidrio se presenta como una excelente alternativa ofreciendo un mejor soporte para rehabilitar la unidad. Conclusión: concluimos que la técnica utilizada aporta beneficios concretos tanto para el profesional como para el paciente. Porque tiene un módulo de elasticidad cercano a la dentina, lo que permite una mejor distribución de las cargas, reduciendo así el riesgo de fracturas.

Palabras clave: Rehabilitación; Pasadores de fibra de vidrio; Estética; Alfileres anatómicos. 


\section{Introdução}

Quando há perda de suporte dentário por diversos fatores como lesões cariosas, traumatismos, procedimentos restauradores feitos anteriormente somado ao desgaste adicional devido a procedimentos endodônticos resulta em perda de suporte dentário (Mezzomo et al., 2002), na maioria dos casos para auxiliar na retenção final da restauração há a necessidade da utilização de retentores intraradiculares. Os pinos de fibra de vidro foram introduzidos na odontologia não só com esse intuito, mas também como uma boa opção estética aos pacientes (Barbosa et al., 2016)

Os pinos de fibra pre fabricados diminuem o tempo de tratamento clínico e possuem o módulo de elasticidade semelhante ao da dentina, um dos critérios utilizados para a seleção do pino de fibra de vidro, é a quantidade de remanescente radícular e o comprimento da raiz (Borges, 2017)

Para os dentes com canais amplos ou raízes fragilizadas, temos uma opção bem viável que são os pinos de fibra de fibro anatômicos, no qual é feito o reembasamento para a melhor adequação do conduto, evitando assim um espaço entre o pino e a parede do canal (Clavijo et al., 2008)

Por demonstrar um desempenho biomecanico satisfatório, um bom resultado estético e elevada aderência aos sistemas cimentantes, os pinos de fibra de vidro tem sua preferência no meio odontológico atual (SILVA et al, 2009), não esquecendo também da sua ótima absorção de cargas mastigatórias, pelo fato da sua estrutura semelhante a dentina, evitando o risco de fraturas radiculares (Tático et al., 2017)

Com a técnica da utilização de pinos de fibra de vidro, além de se devolver a saúde bucal do paciente, devolvemos a função tanto estética como funcional dos dentes, promovendo assim seu bem estar, físico, mental e social, reintegrando-o a sociedade (Ferreira et al., 2016)

O objetivo deste trabalho é abordar os pinos de fibra de vidro, suas vantagens, indicações de uso, visando estabelecer parâmetros clínicos que possam orientar as decisões do profissional ao escolher essa técnica reabilitadora.

\section{Metodologia}

Para a construção desse artigo científico foi utilizada pesquisa de revisão bibliográfica descritiva da literatura. A revisão descritiva propõe a identificação, registro e análise das características, fatores ou variáveis que se relacionam com o fenômeno ou processo (Perovano, 2014). Dessa forma, embasada em referências bibliográficas e científicas, a utilização de pinos de fibra de vidros anatômicos como uma alternativa para a melhoria estética em tratamentos reabilitadores.

A literatura cientifica foi baseada em artigos anexados no Google Acadêmico, SciELO, LILACS e PUBMED com períodos de 23 anos que dizem a respeito sobre pinos de fibra. Pelo fato de haver poucos artigos científicos que abordam sobre dá a utilização de pino de fibra, foi necessário realizar a revisão bibliográficas de artigos antigos. Foram utilizadas as palavras chaves: Reabilitação, Pinos de fibra de vidro, Estética. Pinos anatômicos e o índice glicêmico, biomassa e seu papel na prevenção de doenças. Foi considerado a busca por descrever os benefícios da utilização da utilização de pinos de fibra de vidro.

Os critérios de inclusão deste estudo foram publicações do tipo artigos científicos disponíveis na íntegra e com acesso eletrônico livre, com a abordagem pino de fibra de vidro e seus benefícios relacionados à saúde humana, no qual foi dado prioridade para os estudos em português. 


\section{Resultados e Discussão}

\subsection{Pinos de fibra de vidro}

A utilização de pinos de fibra de vidro dentre os retentores intraradiculares, vem se tornando uma ótima opção, demonstrando resultados satisfatórios e de alta qualidade, devido suas características estéticas e funcionais (Angelus, 2019).

Os pinos são formados por filamentos de fibra de vidro compostos por sílica, alumínio e óxido de magnésio em sua estrutura, combinados longitudinalmente por uma matriz de resina composta, orientaria ao longo do eixo do pino de forma paralela, formando um corpo resistente, sólido, único e bi compatível com o dente (Prado et al., 2014).

\subsection{Indicações}

Os pinos de fibra de vidro são retentores intraradiculares indicados para elementos com extensa destruição coronária, levando em conta sua posição, função, oclusão e estrutura dental remanescente (Costa et al., 2019).

Os dentes tratados endodonticamente, precisam ter suas porções coronárias reconstruídas para que seja restabelecida sua forma e função. A confecção de um núcleo preenchido nesses casos pode oferecer retenção suficiente a coroa dentária (Smitch et al., 1998).

\subsection{Classificação}

Quanto a sua superfície podem ser lisas ou serrilhadas e com formatos variados entre cômicos, paralelos ou paralelo cônico. Podem ter $0,5 \mathrm{~mm}, 1 \mathrm{~mm}, 2 \mathrm{~mm}$ ou $3 \mathrm{~mm}$ de diâmetro, sua escolha vai depender do diâmetro do conduto a ser preparado (Barbosa et al., 2016).

Um dos pontos mais relevantes que influenciam na retenção do pino, é justamente seu formato. Os pinos paralelos apresentam maior retenção e a distribuição de cargas de modo uniforme ao longo do seu eixo, concentrando-as no ápice da raiz, que é duas porções mais estreitas e cônicas. Os cônicos apresentam formato semelhante ao conduto, o que lhe permite menor desgaste da dentina principalmente na porção apical, no entanto possui desvantagens como o efeito cunha na raiz e a concentração de tensão da coroa do dente. E por último o pino paralelo cônico que é o mais indicado em relação ao formato, por possuir um formato mais anatômico, paralelo na região coronária e cônico na região apical, reduzindo o desgaste da dentina e apresentando maior retenção na área cervical devido seu formato paralelo (Barbosa et al., 2016).

\subsection{Vantagens}

Nos tempos de hoje, dentre os retentores intraradiculares, não só como em restaurações estéticas, o desejo pela estética vem sendo cada vez mais procurado por pacientes, os pinos de fibra de vidro foram introduzidos na odontologia, justamente buscando isso, o que os pinos metálicos deixavam a desejar (Barreto et al., 2016).

Devido a sua praticidade, baixo custo, ótimo resultado estético e translucidez, que favorece a cimentação adesiva, além do módulo de elasticidade ser semelhante ao da dentina, favorecendo a distribuições de tensões ao longo da raiz e consequentemente evitando fraturas, os pinos de fibra de vidro são considerados os mais escolhidos entre os cirurgiões dentistas (Mazaro et al., 2014).

Esse pino vem sendo cada dia mais indicados dentro da odontologia, por fornecerem um ótimo desempenho mecânico, resultado estético satisfatório e mostram também uma boa adesão sou sistemas cimentantes. (Silva et al., 2009).

\subsection{Técnicas}

Com intuito de melhorar a adaptação dos pinos de fibra de vidro pré-fabricados nas paredes do conduto radicular e tornar a camada de cimento mais fina, surgiu a técnica de pino de vidro anatômico (Pereira et al., 2011), que consiste na ré 
embasamento dos pinos de fibra de vidro associada a resina composta, desta forma, é realizada a modelagem do conduto com resina fotoativa aderida ao pino, confeccionando pinos individualizados (Grandini et al., 2013).

A individualização do pino garante uma melhor adaptação só canal radicular, permitindo uma camada de cimento resinoso mais fina, favorecendo o aumento da retenção (Kuga et al., 2014).

Os pinos de fibra pré fabricados possuem características semelhantes à da dentina, excelente estética, ótima resistência e associada a técnica de ré embasamento com resina composta sem se mostrando bastante eficaz e acessível. (Barbosa et al., 2016).

Para ser realizada a confecção do pino anatômico pode-se realizar de três maneiras diferentes;

1. Técnica direta: O pino é ré embasamento diretamente no canal (Corrêa et al., 2017).

2. Técnica indireta: Faz-se uma moldagem prévia do canal no modelo de gesso (Corrêa et al., 2017).

3. Técnica semidireta: $\mathrm{O}$ conduto é moldado com silicone e a confecção do pino se faz no próprio molde. (Corrêa et al., 2017).

A técnica direta pode ser executada logo após o tratamento endodôntico, em uma única sessão. Após a desobturação e preparo do conduto, faz-se a lubrificação com glicerina, é feita a inserção do pino juntamente com a resina composta e por último foto polimerizado por 40s (Boing et al., 2011; Guiotti et al., 2014).

Na técnica indireta, após o preparo do conduto, é inserido um pino dura Lay, criando uma retenção adicional, faz-se a moldagem com um material de melhor precisão para se obter um molde preciso e sem distorção, no caso o elastômero, após isso existe uma etapa laboratorial, na qual o molde será vazado no gesso, com o molde de gesso preparado, faz-se a seleção do pino conforme o tamanho do conduto. O canal radicular do modelo é lubrificado com glicerina, logo após é inserido a resina composto junto ao pino e foto polimerizados por $40 \mathrm{seg}$. uma desvantagem dessa técnica, é esse tempo adicional da etapa laboratorial, levando mais de uma sessão para ser feito o procedimento, diferentes das outras técnicas. (Camarinha et al., 2008).

Na técnica semidireta, é feita a desobturação com broca Largo, depois a lubrificação com glicerina e após isso o pino pré fabricado com resina dura Lay é colocado no conduto, com o intuito de criar uma retenção adicional e auxiliar na retirada do material dentro do conduto. Então é feita a moldagem com silicone de condensação, em seguida é feita a duplicação, com o silicone de adição. Com o modelo de silicone pronto, é inserido a resina composta dentro do conduto e em seguida feita a adaptação do pino do mesmo, por último foto polimerizando por $40 \mathrm{seg}$. O pino pode ser cimentado logo após sua individualização, em uma única sessão (Corrêa et al., 2017).

Todas essas técnicas tem em comum adaptar-se perfeitamente ao conduto radicular. O material de ré embasamento, no caso a resina composta junto ao pino de fibra de vidro, se adaptam perfeitamente as paredes do canal, resultando em uma cimentação uniforme, favorecendo a retenção do pino no canal (Teixeira et al., 2009).

\subsection{Protocolo clínico}

Para receber um retentor intraradicular o dente precisa ser preparado, deve-se então remover parcialmente parte do material obturador, deixando no mínimo $3 \mathrm{~mm}$ ou mais para não comprometer o sucesso do tratamento endodôntico, deslocamento da obturação e evitar qualquer tipo de infiltração (Shilling Burg et al., 1998, Miranda et al., 2000, Heling et al., 2002), um dos pontos mais importantes para se manter a resistência a fratura do dente a ser preparado é a espessura de dentina remanescente, então durante esse preparo deve ser preservado ao máximo a estrutura dentária do conduto.(Shiling Burg, Kessler et al., 1991)

Segundo Pereira (2011), Clavijo \& Kabbach (2014) os protocolos clínicos para colocação do pino anatômico de fibra de vidro são: 
1. Desobturação do conduto: com o uso de calcadores de Paiva, seguidos da broca Largo \& Gattes.

2. Seleção de pino: Faz-se a seleção do pino de acordo com a largura e altura do conduto. A utilização de radiografias periapicais são indicadas para cervical a adaptação cervical.

3. Preparo do conduto: as paredes internas do conditos devem ser regularizadas com os sistemas de brocas do pino sempre fabricado a ser utilizado.

4. Condicionamento do pino - Ataque ácido com ácido fosfórico 37\% por 60 segundos, lavagem com água corrente por 30 segundos, secagem, aplicação do Silano por um minuto, aplicação do sistema adesivo, depois faz-se a remoção de excessos com jato de ar e foto polimerizado por 40 segundos.

5. Seleção da resina para ré embasamento - a resina a ser escolhida deve conter boas propriedades mecânicas, opaca com cromaticidade compatível com a coloração dos dentes homólogos com o intuito de impedir que os núcleos de preenchimento se tornem acinzentados, causado geralmente pelo uso de resina translúcidas.

6. Ré embasamento do pino de acordo com a técnica a ser escolhida.

7. Condicionamento do pino anatômico - Deve-se fazer o tratamento da superfície com ácido fosfórico 37\%, lavar com água, secar, aplicar uma fina camada de adesivo, remoção de excessos com o jato de ar, foto polimerização por 20 segundos. Devido ao fato de o pino anatômico estar intimamente adaptado ao conduto, essa etapa exige cautela, pois qualquer excesso de material pode comprometer sua adaptação.

8. Cimentação do pino anatômico - é recomendado a utilização de cimentos resinosos autoadesivos ou químicos. (Pereira, 2011; Clavijo \& Kabbach,2014).

Para apresentar uma boa resistência, a camada de cimentação deve ser fina e uniforme, evitando a formação de porosidades e reduzindo as tensões de contrações geradas, portanto o procedimento de inserção de cimento deve ser feito de maneira cuidadosa, evitando assim o enfraquecimento desse elo de união. (Watzke et al., 2008; Sausen, 2011).

\subsection{Tipos de cimentos resinosos}

Os cimentos resinosos podem ser divididos em três tipos, de acordo com a reação de polimerização (Weiser \& Behr, 2015)

1. Quimicamente ativos (auto polimerizados)

2. Fisicamente ativos (fotoativos)

\subsection{Dupla polimerização (dual)}

O cimento dual é o que mais se destaca em relação aos outros, e pode ser classificado como autoadesivo e convencional. Diferente dos cimentos convencionais, os cimentos resinosos autoadesivos não necessitam de pré-tratamento da superfície dentária, por conta da sua composição que associa o uso do adesivo e do ácido. (Weiser \& Behr, 2015).

\subsection{Fatores prejudiciais à união pino/dentina}

Apesar da técnica dos pinos anatômicos apresentar alto percentual de sucesso, alguns fatores podem alterar de forma negativa a união entre os pinos de fibra de vidro e a dentina radicular, tais como (Carvalho et al., 2016)

1. A contração de polimerização do cimento resinoso no canal radicular (Carvalho et al., 2016)

2. A dificuldade de polimerização do cimento dentro do conduto (Carvalho et al., 2016

3. A forma dos canais (Carvalho et al., 2016)

4. Qualidade e quantidade da dentina remanescente (Carvalho et al., 2016) 


\section{Discussão}

Segundo Terry e Geller et al., (2014) os pinos metálicos são fundidos numa liga com módulo de elasticidade bem maior que o da dentina, esta incompatibilidade com a transmissão de forças oclusos facilita a fratura radicular

Pegoraro et al. (2004) afirma que com o uso dos pinos de fibra de vidro, pode -se obter ótimos resultados estéticos, além de inúmeras vantagens, suas propriedades estruturais similares a dentina, permitem a distribuição de tensões, suportando próteses ou restaurações, evitando a fratura de raiz. Pereira et al., (2011) cita também que um dos benefícios quanto a utilização dessa técnica, é a diminuição dos números de consultas, por conta de sua praticidade, sendo assim uma ótima alternativa clínica a dentes desvitalizados., Clavijo et al., (2014) menciona quanto a vida longa de restaurações e a aparência similar ao dente natural na utilização dessa técnica.

Para Prado et al., (2014), os pinos formam um corpo resistente, sólido, único e bi compatível devido a sua estrutura formada por filamentos de fibra de vidro, compostos por sílica, alumínio e óxido de magnésio, juntamente por uma matriz de resina composta, orientadas ao longo do pino de forma paralela.

Para Baratieri et al., (2004); Silva et al., (2009) as características ideais de um pino intraradicular devem ser: bi compatíveis, possuir propriedades estruturais semelhantes aos tecidos dentais, fornecer um bom desempenho mecânico, uma boa aderência aos sistemas cimentantes, permitir a distribuição de tensões, a fim de evitar fraturas radiculares.

Souza, Junior et al., (2012) sugeriram a utilização da técnica de pinos anatômicos com resina composta, para aqueles casos em que há

pouco remanescente coronário para suporte da restauração e para canais amplos, afim de diminuir a linha de cimentação.

Para Monte alto et al. 2016 e Pereira et al 2011, com essa técnica pode-se alcançar melhor adaptação com as paredes do canal radícular, produzindo uma fina camada de cimento o que implica diretamente com o desempenho clínico da técnica. Chieffi et al., 2007, salienta que o uso de pinos anatômicos exerce pressão de assentamento sobre o cimento contra as paredes do canal, fazendo com que penetrem mais no substrato.

Pedrosa-Filho (2006) comparou pinos pré fabricados com os pinos no qual foram feitos a individualização, os resultados mostraram que os pinos de fibra de vidro reembasados atingiram valores de resistência de união mais elevados do que os apenas cimentados, dessa forma se mostrando eficaz para elevar a retenção de pinos de fibra de vidro.

Contudo, a técnica de ré embasamento dos pinos de fibra de vidro. tem demonstrado ótimos resultados em relação a resistência de união, além de ter fácil execução e poder ser realizada clinicamente. (Velmurugan \& Parameswaran, 2004; Iglesias-Puig \& Arellano-Carbonero, 2004; Tanoue et al., 2007), tem sido bastante efetiva em diferentes situações clínicas, sendo assim considerada uma nova opção de conservação de estrutura dental (Tanoue et al., 2007).

\section{Conclusão}

Com relação ao presente trabalho apresentado, concluímos que a técnica utilizada traz benefícios concretos tanto para o profissional quanto para o paciente. Por possuir o modulo de elasticidade próximo a dentina, possibilitando assim uma melhor distribuição de cargas, reduzindo assim o risco de fraturas.

Sendo assim cabe ao Dentista está sempre se atualizando e conhecendo as técnicas, as propriedades do material e suas limitações, para que possa aplicar de uma maneira mais eficaz de forma a obter melhores resultados.

\section{Referências}

Angelus. Pinos de Fibra de Vidro: Entenda as Principais Vantagens de Usar. blog.angelus.ind.br/pino-de-fibra-de-vidro/>.

Angelus. Splendo SAP: Angelus. <angelus.ind.br/produto/splendor-sap/>. 
Baratieri, L. N. (2001). Odontologia Restauradora Fundamentos e Possibilidades. Santos, 739.

Barbosa, I. F., et al. (2016). Pinos de fibra: revisão de literatura. Revista Uningá Review. 28(1),83-87.

Barbosa, I. F., Barreto, B. C. T., Coelho, M. D. O., Pereira, G. D. D. S., \& de Carvalho, Z. M. C. (2016). Pinos de fibra: revisão da literatura. Rev Uningá Review, 28(1), 83-7.

Boing, T. F et al. (2011). Avaliação do grau de conversão de uma resina composta utilizando diferentes tratamentos de superfície previamente à fotopolimerização final. Rev Dent online. 22, 9-14. http://coral.ufsm.br/dentisticaonline/1018.pdf.

Camarinha, S. M. L. B. (2008). Avaliação da adaptação de núcleo metálico fundido em função da técnica de moldagem. Dissertação (Mestrado em Reabilitação Oral). Faculdade de Odontologia de Ribeirão Preto, Universidade de São Paulo.

Chieffi, N., et al. (2007). The effect of application sustained seating pressure on adhesive luting procedure. Dent Mater, 23(2), 159-64.

Clavijo, Victor., \& Kabbach, W. (2014). Pinos anatômicos: acredite nessa técnica. Clínica-International Journal of Brazilian Dentistry, Florianópolis, 10(1), 12- 21 .

Corrêa, L. R. N., et al. (2017). Confecção de pino anatômico pela técnica semidireta. Revista rede de cuidados em saúde. 10(1). http://publicacoes.unigranrio.edu.br/index.php/rcs/article/view/4281/2240

Costa, D. D., Carvalho, L. C. B., Nery, F. S., Barbosa, P. A., Amado, D. S., \& Cruz, J. S. W. (2011). Avaliação quantitativa do remanescente dentinário após instalação de pino de fibra de vidro em incisivos inferiores com raízes achatadas. Revista de Ciências Médicas e Biológicas, 10(1), 39-43. Salvador - BA.

Guiotti, F. A., Guiotti, A. M., Andrade, M. F., \& Kuga, M. C. Visão Contemporânea Sobre Pinos Anatômicos, Arch Health Invest., $2(3), .64-73,2014$.

Iglesia-Puig, Arellano-Carbonero. Fiber-reinforced post and core adapted.

Mazaro, J. V. Q., Santos, A. B., Zavanelli, A. C., Mello, C. C., Lemos, C. A. A., \& Filho, H. G. (2014). Avaliação dos Fatores Críticos para Seleção e Aplicação Clínica dos Pinos de Fibra- Relato de Caso. Revista Odontológica de Araçatuba, 35(2), 26-36.

Mezzomo, E. (2002). Prótese fixa contemporânea. Santos.

Pegoraro, L, F., et al. (2013). Prótese Fixa: Bases Para o Planejamento em Reabilitação Oral. (2a ed), Editora Artes Médicas.

Pereira, Jeferson Ricardo. (2011). Retentores Intra-radiculares. Artes Médicas.

Prado, M, A, A., et al. (2014). Retentores Intrarradiculares: Revisão de Literatura. UNOPAR Científica, Ciências Biológicas e da Saúde. 16(1), 51-55.

Sausen, B. P. (2011). Avaliação em mev da fenda de contração de polimerização entre pino de fibra de vidro e resina composta de baixa contração. 37 f. TCC (Graduação) - Curso de Odontologia, Universidade Federal do Rio Grande do Sul, Porto Alegre.

Scotti, R., \& Ferrari, M. (2003). Pinos de Fibra: considerações teóricas e aplicações clínicas. Artes Médicas.

Shillingburg, H. T., Hobo, S., Whitsett, L. D., Jacobi, R., \& Brackett, S. E. (1998). Fundamentos de prótese fixa. (3rd ed.) Quintessence.

Shillingburg H. T., \& Kessler J. C. (1991). Restauração de dentes tratados endodonticamente. Quintessence Publishing.

Silva, N. R. et al. (2009). Influ.ence of Different Post Design and Composition on Stress Distribution in Maxillary Central Incisor: Finite Element Analysis, Indian J Dent Res. 20, 2, 153-8.

Smith, C. T., Schuman, N. J., \& Wasson, W. (1998). Biomechanica criteria for evaluating prefabricated post-and-core systems: A guide for the restorative dentist. Quintes- sence Int., 29(5), 305-12.

Tanoue., N., et al. (2007). Application of a preimpregnated fiber-reinforced composite in the fabrication of an indirect dowelcore. $J$ Oral Sci. 49, 179-182. https://www.ncbi.nlm.nih.gov/pubmed/17634733.

Teixeira, S. C., et al. (2009). Bond Strength of Fiber Posts to Weakened Roots After Resin Restoration With Different Light-Curing Times. JOE. 35(7), https://www.jendodon.com/article/S0099-2399(09)00362-8/fulltext.

Terry, D. A., Geller, W. (2014). Odontologia estética e restauradora. (2a ed.), Editora Quintessence.

Velmurugan, N. (2004). Parameswaran A. Custom-made resin post and core. Oper Dent. 29(1),112-4.

Watzke, R., et al. (2008). Interface homogeneity of adhesively luted glass fiber posts. Dent. Mater., Kidlington, 24(11), 1512-1517, https://www.ncbi.nlm.nih.gov/pubmed/18466965.

Weiser, F., \& Behr, M. (2015). Self-adhesive resin cements: a clinical review. J Prosthodont. Feb,24(2), 100-8. https://www.ncbi.nlm.nih.gov/pubmed/25041496. 